\title{
DEVELOPMENTS IN NMR IN LIQUID CRYSTALLINE SOLVENTS
}

\author{
Saul Meiboom, Richard C. Hewitt and Lawrence C. Snyder
}

Bell Telephone Laboratories, Inc., Murray Hill, New Jersey 07974.U.S.A.

\begin{abstract}
This paper describes some recent developments in the technique of molecular structure determination from $\mathrm{nmr}$ in liquid crystalline solvents. 1. An INDOR double resonance method, which can be helpful in the analysis of complex spectra, is described and applied to the spectrum of bicyclobutane. 2 . In more complex molecules, extensive overlap of peaks can make interpretation impossible. It is shown that deuterium substitution will greatly simplify the spectra. Decoupling of the deuterium is achieved by irradiation of a double quantum transition. Spectra for cyclohexane are presented. 3. A very simple model for estimating corrections for molecular vibrations is presented. In this model, only the vibrations of the hydrogen atoms, relative to a rigid framework of the heavier atoms, are taken into account. 4. Ditchfield and Snyder have made $a b$ initio molecular orbital calculations for the anisotropy of the indirect couplings. Their principal results for methyl fluoride are presented. It is concluded that in many cases corrections for these couplings are negligible.
\end{abstract}

\section{INTRODUCTION}

The technique of molecular structure determination from high resolution $\mathrm{nmr}$ in liquid crystalline solvents was introduced by Saupe and Englert in a 1963 paper $^{1}$. The first of these authors has reviewed the subject at the previous Conference ${ }^{2}$, and other recent reviews have also appeared ${ }^{3,4}$. Rather than going over the subject again, we shall in the following describe some newer developments in the technique.

Three main difficulties stand in the way of a wider application of the method. The first is that the spectra become rapidly more complex as the number of magnetic nuclei increases. For this reason hardly any molecules with more than eight magnetic nuclei have been studied. Even for such a number, complexity can make the theoretical interpretation of the spectra impractical. For molecules with a larger number of magnetic nuclei, extensive overlap of the multitude of peaks makes interpretation impossible. A second difficulty is the possible inaccuracy of the derived molecular geometry due to molecular vibrations. Finally, a third problem is posed by the anisotropy in the indirect nuclear couplings, which too can be a source of errors.

In the next two sections we shall discuss two techniques which we have found useful in extending the range of applicability of the method. This will be followed by some remarks on the influence of vibrations and the anisotropy of the indirect coupling. Although we cannot claim that we have solved the problems mentioned above, we feel that some progress has been made. 


\section{THE INDOR TECHNIQUE AND THE SPECTRUM OF BICYCLOBUTANE}

The complexity of a spectrum depends of course not only on the number of magnetic nuclei in the molecule (which determines the number of spin states), but also on the degree of symmetry of the molecule (which determines the degree of degeneracy of the states). This is illustrated in Figures 1 and 2, where the first gives the well-known proton spectrum of benzene, and the second the spectrum of bicylobutane. Both molecules contain six protons, but the degree of spectral complexity is obviously very different. The benzene spectrum is readily interpreted on a trial and error basis, taking the single orientational constant and the direct interactions as adjustable parameters.

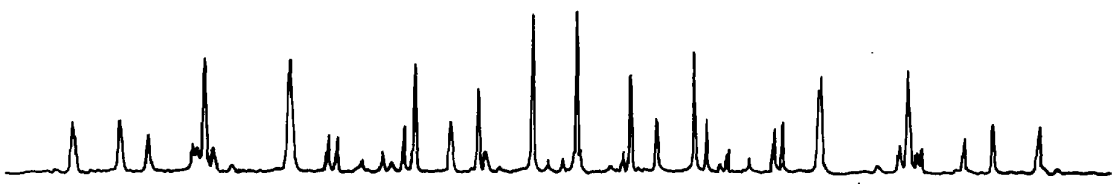

Figure 1. Spectrum of benzene in nematic $4,4^{\prime}-\mathrm{di}(n-\mathrm{h}$ exyloxy)azoxybenzene

On the other hand, the bicyclobutane molecule, assuming that it possesses two planes of symmetry, requires five geometric parameters to specify the relative positions of its protons, two orientational constants to specify the alignment in the anisotropic solvent, and two relative chemical shifts. Even though only ratios of the geometric parameters determine the relative line positions in a spectrum, one is still left with eight parameters to be adjusted when fitting a computer-simulated spectrum with the observed one. Even with a reasonable idea as to the geometry of the molecule, we did not succeed in finding a satisfactory set of parameters by a simple trial and error method, though over one hundred trial Hamiltonians were computed. It is not difficult to find a set of parameters which reproduces the overall aspect of the spectrum. However, attempts to refine the simulations by varying the parameters failed, because the multitude of peaks made the establishment of a one-to-one correspondence between calculated and observed lines impossible, and as a result it could not be decided whether a change in parameter improved or degraded the fit.
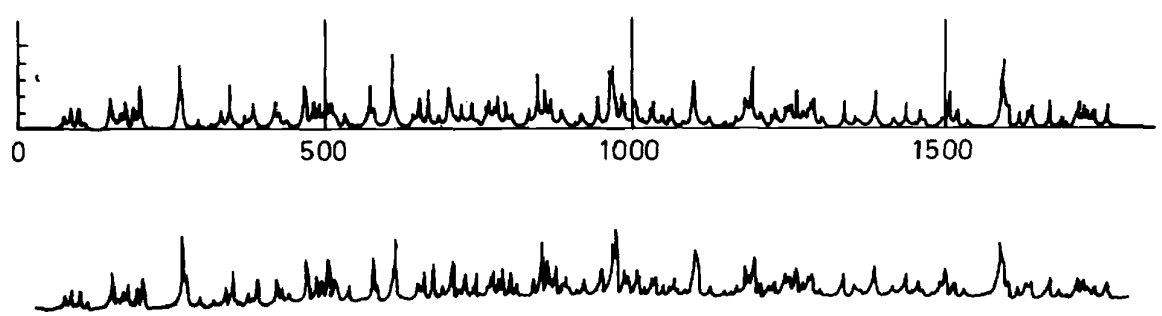

Figure 2. Bottom trace: experimental spectrum; and top trace: simulated spectrum of bicyclobutane. The scale is in hertz 
The use of a proton-proton INDOR technique solved the problem. In this well-known double irradiation technique, one transmitter, as well as the receiver, are set to the centre frequency of a selected spectrum peak, and the response of this peak recorded when sweeping a second, partially saturating, transmitter frequency through the spectrum. The actual spectrometer used had a time division scheme for the observation and irradiation frequencies, which eliminated disturbing beat frequencies. It has been described previously ${ }^{5}$. For the present purpose it is sufficient to consider only

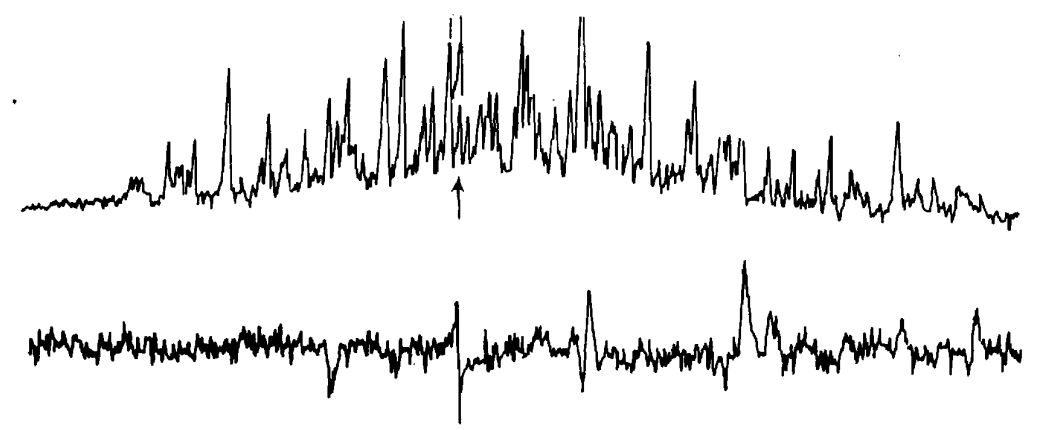

Figure 3. The bottom trace shows an observed INDOR spectrum of bicyclobutane in a nematic solvent. The top trace shows the regular nmr spectrum on the same frequency scale. (The fall-off in signal intensity in the wings of this spectrum is instrumental.) The arrow indicates the peak observed during the INDOR record

the population changes in the levels resulting from the passage of the second, saturating, transmitter frequency through the spectrum. Only passage through those peaks that have a level in common with the observed peak will result in a response in the receiver output. This response will be positive if, in the energy level scheme, the irradiated transition is either above or below the observed one, as in this case the population difference of the observed transition is increased. The response will be negative if the irradiated transition involves levels that are at nearly the same energy (that is the same $I_{z}$ values) as the levels of the observed transition.

An example of an observed INDOR spectrum is given in Figure 3, and a computer-simulated spectrum in Figure 4. A useful way to look at the simplification obtained in the INDOR spectra is to consider the latter as subspectra of the regular spectrum. In the case of bicyclobutane, the molecule has two planes of reflection symmetry, one containing the bridgehead carbon atoms, and the other the methylene carbon atoms. The spin Hamiltonian is accordingly invariant for interchange of the corresponding protons, and the wavefunctions can be classified into four sets: even-even, even-odd, odd-even and odd-odd under the two symmetry operations. Transitions are allowed only between states belonging to the same set, and thus the spectrum can be considered to consist of four independent subspectra, which can be "pulled out' by the INDOR technique and compared with the corresponding calculated subspectra. Of course, a complete classification of the lines is not 
necessary. As one can work with an INDOR spectrum containing relatively few lines, it is now possible to establish a one-to-one correspondenoe with a calculated spectrum, and adjust the parameters of it for a fit. Once a reasonable approximation is obtained, line assignments in the complete spectrum can be made, and final refinement done with the latter.

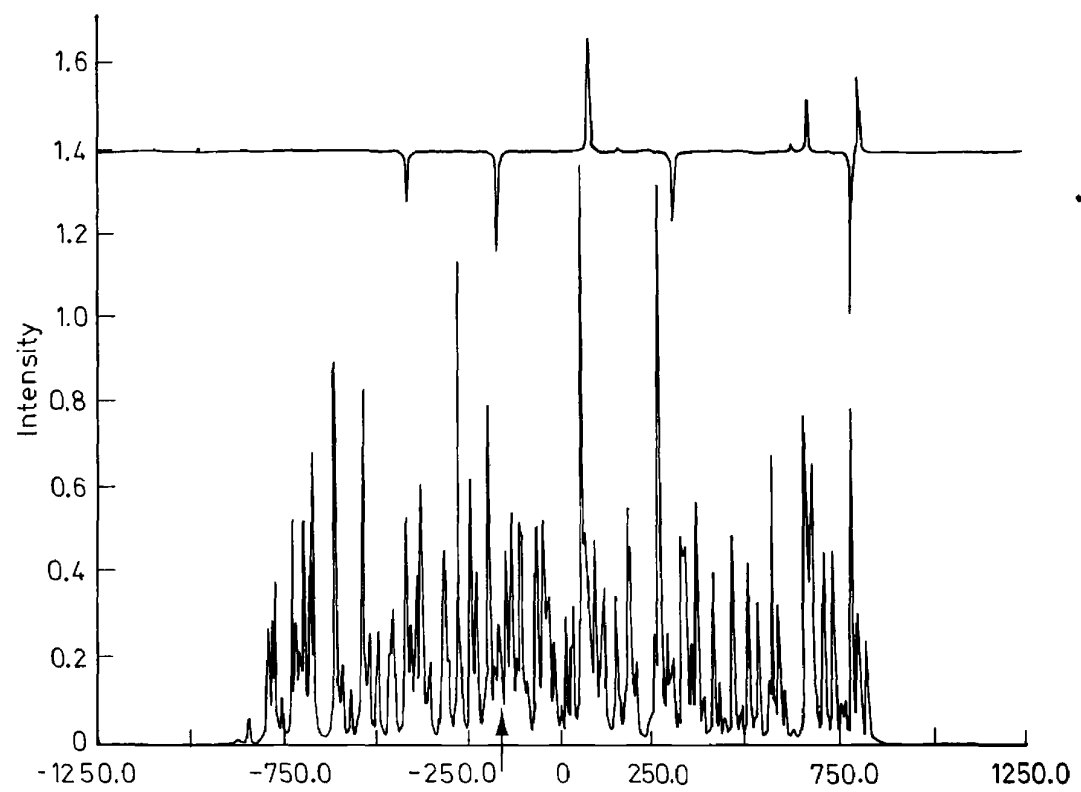

Figure 4. The top trace shows a computer-simulated INDOR spectrum, and the bottom trace, the simulated regular spectrum. The arrow indicates the peak for which the INDOR spectrum was computed

The final simulated spectrum for bicylobutane is given in the top trace of Figure 2. For the molecular geometry derived from this analysis we refer to reference 4.

\section{DEUTERIUM SUBSTITUTION AND THE SPECTRUM OF CYCLOHEXANE}

The proton spectrum of cyclohexane in a nematic solvent is shown in the top trace of Figure 5. Clearly, extensive overlap of peaks occurs, and detailed spectrum interpretation is impossible. Partial deuterium substitution greatly simplifies the spectrum and makes interpretation possible. The spectra to be discussed were taken on cyclohexane- $\mathrm{d}_{11}$, which had a deuterium isotopic purity of 98 per cent. Thus about 80 per cent of the cyclohexane molecules contain a single proton, 18 per cent two protons, and 2 per cent three or more protons. Trace 3 of Figure 5 shows the spectrum of this deuterated cyclohexane. The broad triplet structure is due to the dipolar coupling of the single proton of the dominant species with the deuteron on the same carbon. 


\section{NMR IN LIQUID CRYSTALLINE SOLVENTS}

Coupling with more distant deuterons accounts for most of the width of the component peaks. Decoupling of the deuterons, by irradiation at the deuteron frequency, gives the spectra reproduced in traces 4 and 5 of Figure 5, trace 5 differing from trace 4 only in that the spectrum was recorded at 32 times higher sensitivity. The interpretation of these spectra is straightforward.
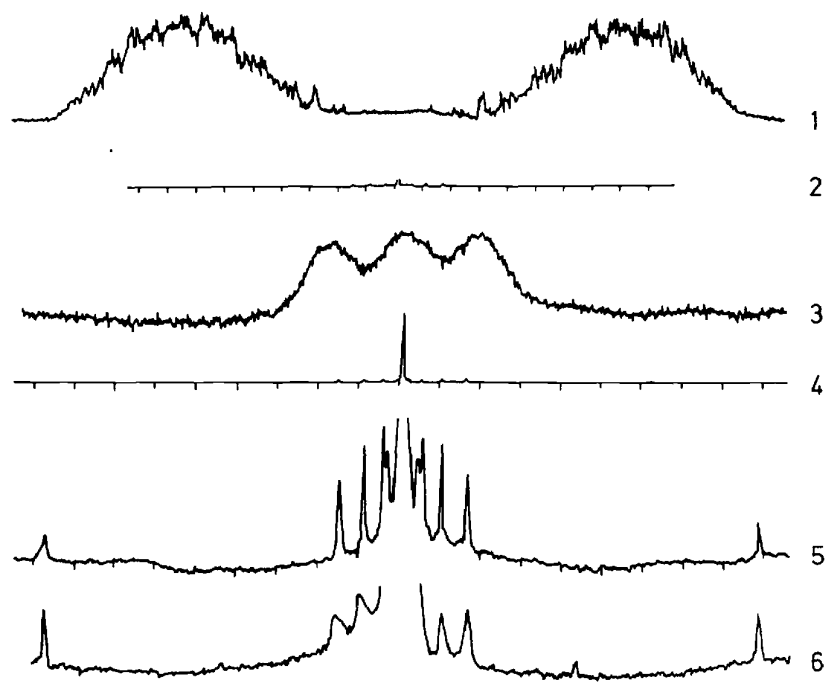

Figure 5. Trace 1: The spectrum of cyclohexane; the frequency scale is given by Trace 2, in which the markers are spaced by $100 \mathrm{~Hz}$. Trace 3: The spectrum of cyclohexane- $\mathrm{d}_{11}$ without deuterium decoupling. Trace 4: The same sample as trace 3 , but with deuterium decoupling and recorded at low senstivity. The frequency markers are $100 \mathrm{~Hz}$ apart and apply to traces 3 through 6 Trace 5: As trace 3, but recorded at 32 times higher sensitivity. Trace 6: As trace 5, but with deuterium irradiation frequency offset by $65 \mathrm{~Hz}$. All spectra were made in 4,4'-bis(hexyloxy)azoxybenzene as the nematic solvent

At the temperature of the experiment $\left(102^{\circ} \mathrm{C}\right)$, the cyclohexane molecule inverts rapidly (on the nmr time scale) between two chair forms for which axial and equatorial protons are interchanged. The observed $\mathrm{nmr}$ frequencies are the averages for these two conformations. Thus one expects a single strong peak for the cyclohexane molecules containing a single proton, and a symmetrical pair of peaks from molecules containing two protons. There are seven such species, each having a different relative position of its protons, and thus a different magnitude of their dipolar interaction and a corresponding spacing of the components of the doublet. The outermost pair of peaks in trace 5 of Figure 5 can be identified as due to the species having two protons on the same carbon atom. The central part of the spectrum is shown in greater detail in trace 1 of Figure 6. Each pair of peaks located symmetrically around the centre can be ascribed to a specific molecular species.

In obtaining the above spectra, effective deuterium decoupling is essential and a description of how this was achieved follows. The problem is complicated by the fact that the deuterium resonance is split into two rather widely 
separated components, due to quadrupole interaction in the anisotropic environment of the liquid crystal. Decoupling by noise modulation proved ineffective at available power levels. It was, however, found that c.w. irradiation at a frequency midway between the two components will induce double quantum transitions between the +1 and -1 deuteron levels, and provides excellent decoupling at reasonable power levels (about 20 watt). It is characteristic of the double quantum transitions that the irradiation frequency is quite critical; a deviation from the exact centre frequency of even a few hertz results in appreciable broadening of the proton peaks. This fact should be kept in mind when using the method. For instance, we were puzzled for
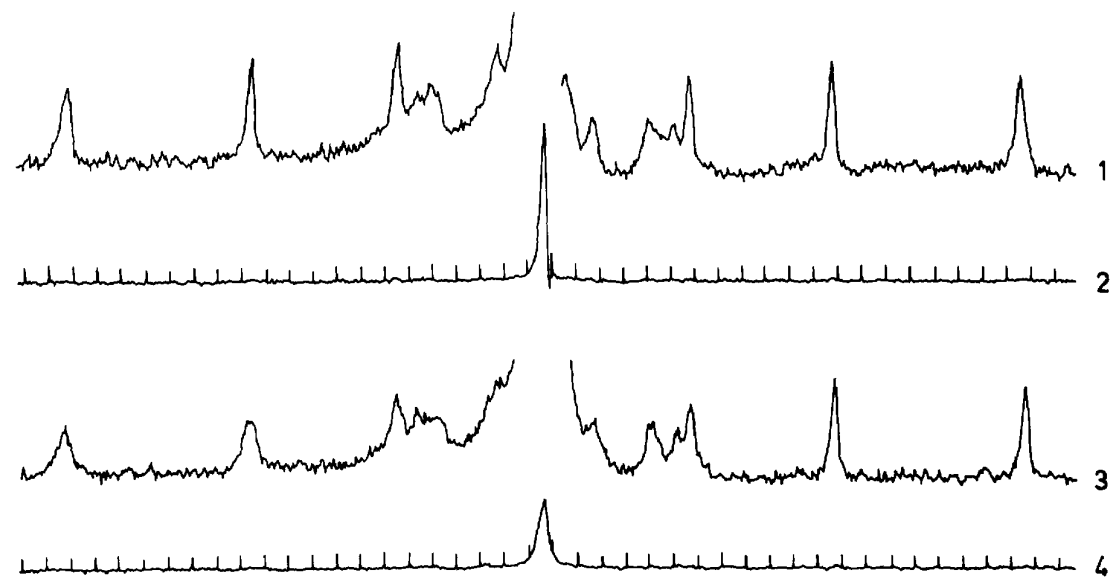

Figure 6. Trace 1: Detail of the central part of the spectrum of cyclohexane- $\mathrm{d}_{11}$. Trace 2: As trace 1, but with reduced sensitivity to show central peak. The frequency markers are $10 \mathrm{~Hz}$ apart. Trace 3: As trace 1, but with deuterium irradiation frequency offset by $10 \mathrm{~Hz}$. Trace 4: As trace 3, but with reduced sensitivity

some time as to why peaks due to molecules containing one ${ }^{13} \mathrm{C}$ nucleus did not show up in the spectrum reproduced as trace 5 of Figure 5. One can easily calculate that for molecules containing one proton bound to a ${ }^{13} \mathrm{C}$ in natural abundance ( 1 per cent), the intensity of the corresponding peaks should be about one quarter of those of the outermost peaks in trace 5 . In fact, no such peaks could be detected. It was then realized that the ${ }^{13} \mathrm{C}$ nucleus couples to the deuteron as well as to the proton, and thus the deuteron frequency is shifted to higher or lower frequency, depending on the spin state of the ${ }^{13} \mathrm{C}$. Indeed, when the deuterium frequency was offset by $65 \mathrm{~Hz}$, the peak due to the ${ }^{13} \mathrm{C}$ molecular species did appear, as illustrated in trace 6 of Figure 5. Of course, other peaks are now broadened, because the deuterium irradiation is off resonance for them. Note that the outermost peaks, due to molecules with gem protons, do not broaden appreciably, because the deuterons are farther from the protons here, and the resulting smaller coupling is more easily averaged out.

A similar situation exists in the species containing two protons. Here too the deuterons gem to the protons are most critical to decouple because of their 
large interaction. But the frequency of such a deuteron is shifted by the dipolar coupling according to the spin state of the far proton. That this is indeed the case can be seen in trace 3 of Figure 6, which was recorded with the deuterium irradiation frequency offset by $10 \mathrm{~Hz}$. The second peak from the right has become narrower, but most other peaks are broader compared with trace 1. One can thus correlate a proton-deuteron interaction with a protonproton interaction, and if the deuteron offset frequency is converted into proton frequency, one can correlate the peaks of a species in which the two protons are in the cis relation to the peaks of the corresponding species in which the protons are in the trans relation. A systematic study of line narrowing as a function of deuterium frequency offset can thus be a help in assigning the spectrum lines.

The objection may be made that the above reasoning, while perfectly valid for the ${ }^{13} \mathrm{C}$ case, is inapplicable for the two-proton case, because both protons are equivalent, their eigenstates mixed, and thus the effective field picture to describe the coupling of a single proton with a deuteron may not be a reasonable approximation. For this reason a more detailed theoretical investigation was undertaken. The simple theory for treating double irradiation given by Bloom and Shoolery ${ }^{6}$ was extended to include quadrupole interactions and direct dipolar interactions; it was also generalized to apply to more than two nuclei. The spin Hamiltonian in the frame rotating with the deuterium irradiation frequency can be written (in frequency units) as:

$$
\begin{gathered}
\mathscr{H}=\sum_{d}\left[-\left(v_{0 d}-v_{2}+\delta_{d}\right) I_{z d}-v_{2 d} I_{x d}+A_{d}\left(3 I_{z d}^{2}-2\right)\right] \\
+\sum_{p}\left[-\left(v_{0 p}-v_{2}+\delta_{p}\right) I_{z p}\right] \\
+\sum_{d} \sum_{p}\left(J_{d p}+D_{d p}\right) I_{z d} I_{z p} \\
+\sum_{p^{\prime}>p} J_{p p^{\prime}}\left[I_{z p} I_{z p^{\prime}}+\frac{1}{2}\left(I_{+p} I_{-p^{\prime}}+I_{-p} I_{+p^{\prime}}\right)\right] \\
+\sum_{p^{\prime}>p} D_{p p^{\prime}}\left[I_{z p} I_{z p^{\prime}}-\frac{1}{4}\left(I_{+p} I_{-p^{\prime}}+I_{-p} I_{+p^{\prime}}\right)\right] \\
+\sum_{d^{\prime}>d} J_{d d^{\prime}}\left[I_{z d} I_{z d^{\prime}}+\frac{1}{2}\left(I_{+d^{\prime}} I_{-d^{\prime}}+I_{-d} I_{+d^{\prime}}\right)\right] \\
+\sum_{d^{\prime}>d} D_{d d^{\prime}}\left[I_{z d^{\prime}} I_{z d^{\prime}}-\frac{1}{4}\left(I_{+d} I_{-d^{\prime}}+I_{-d_{d}} I_{+d^{\prime}}\right)\right] \\
v_{0 d}=\frac{\gamma_{d} H_{0}}{2 \pi}, \quad v_{0 p}=\frac{\gamma_{p} H_{0}}{2 \pi} \\
v_{2 d}=\frac{\gamma_{d} H_{2}}{2 \pi} \\
A_{d}=\frac{e^{2} q Q}{4 h}\left\langle\frac{3 \cos ^{2} \theta-1}{2}\right\rangle
\end{gathered}
$$

Here

In these equations the subscript $d$ refers to the deuterons, $p$ to the protons, $H_{0}$ is the magnitude of the constant magnetic field along the $z$-axis, $H_{2}$ the 
magnitude of the rotating component of the deuterium irradiation field, $v_{2}$ the frequency of that field, $\delta$ the chemical shifts, $J$ the magnitude of the scalar (indirect) coupling, $D$ the magnitude of the dipolar (direct) coupling. The other symbols have their conventional meaning.

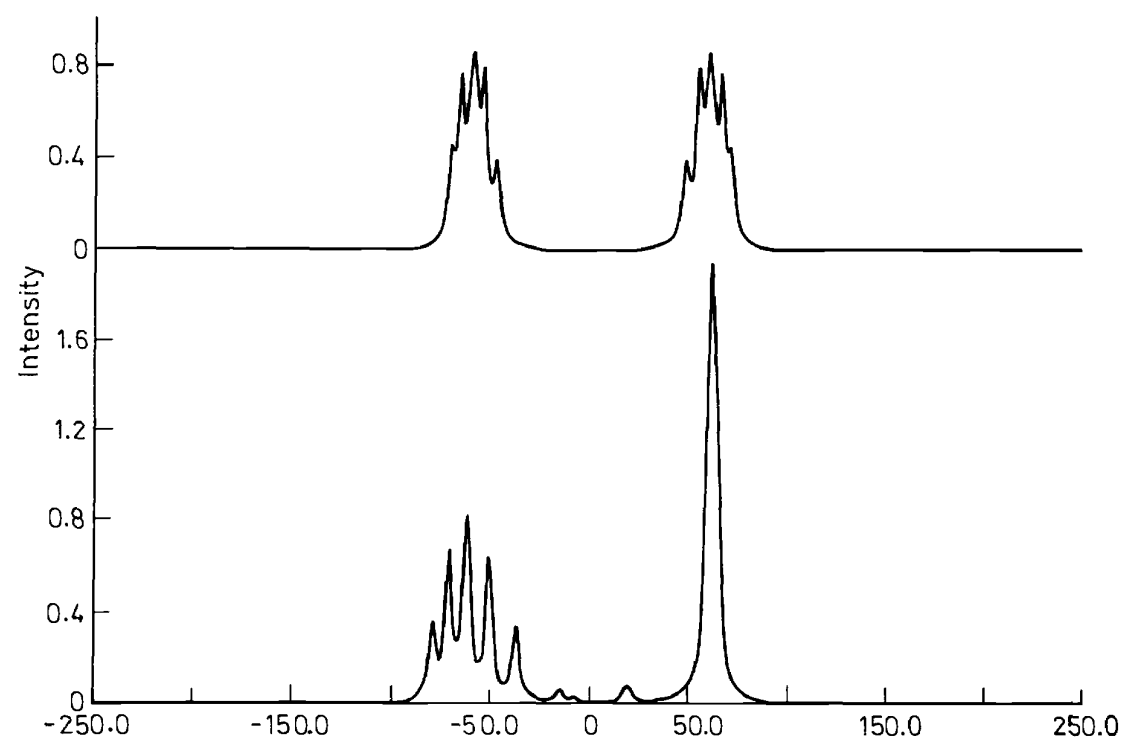

Figure 7. Computer simulated proton spectra for two protons and two deuterons. In the top trace, the deuteron decoupling frequency has zero offset. In the bottom trace the offset is $42 \mathrm{~Hz}$

A computer program was written to simulate spectra based on this Hamiltonian. Figure 7 shows two outputs from this program, giving the proton spectra for the case of two protons and two deuterons. The dipolar coupling between the two protons was taken as $D_{p p^{\prime}}=-81.0 \mathrm{~Hz}$, between a deuteron and its nearer proton $D_{d p}=+228.5 \mathrm{~Hz}$, between a deuteron and its farther proton $D_{d p^{\prime}}=-41.4 \mathrm{~Hz}$. The interaction between deuterons, all the scalar interactions and the chemical shifts were taken as zero. Other parameters were: $A_{d}=-1100 \mathrm{~Hz}$ and $v_{2 d}=2350 \mathrm{~Hz}$. A Lorentzian lineshape with a full width at half height of $4 \mathrm{~Hz}$ was used in the simulation. The top trace of Figure 7 shows the proton spectrum for zero offset of the deuterium frequency, $v_{2}=v_{0 d}$. Each of the components of the doublet is split into a number of lines, giving in effect a broadened peak, due to the fact that the deuterons are off-resonance. The bottom trace shows the spectrum for an offset $v_{2}-v_{0 d}=42 \mathrm{~Hz}$. The collapse of one of the components is obvious. An offset of opposite sign will of course result in a narrowing of the other component. Note that the offset for maximum narrowing is very nearly equal to the $D_{d p^{\prime}}$ interaction, twice that predicted by the naive argument given above. This has proved true for a wide range of parameters, and the effective field picture thus seems to be in error by a factor of two. 
Using the techniques described in this section, a detailed analysis of the cyclohexane spectrum is nearly completed. However, at the time of this writing, final results for the molecular geometry are not yet available.

\section{THE EFFECT OF VIBRATIONS}

The need to correct for molecular vibrations in order to obtain an accurate molecular geometry has been recognized by most authors. In short, the dipolar couplings obtained from a spectrum interpretation are averages over the vibrational motions of the molecule. Because of the non-linear character of the dipolar coupling, these average values do not correspond to the dipolar couplings in the equilibrium state of the molecule. Even if the normal modes of the molecular vibrations were known, an accurate calculation would be complicated by anharmonicity of the vibrations and the unknown perturbations in the liquid state.

Table 1. Comparison of measured and calculated dipolar coupling constants in benzene.

\begin{tabular}{lcccc}
\hline Coupling & $\begin{array}{c}\text { Meas. } \\
\text { Hertz. }\end{array}$ & $\begin{array}{c}\text { Calc. } \\
\text { Hertz. }\end{array}$ & $\begin{array}{c}\text { Difference } \\
\%\end{array}$ & $\begin{array}{c}\text { Vibr. corr. } \\
\%\end{array}$ \\
\hline HH ortho & -964.7 & $(-964.7)$ & - & - \\
HH meta & -186.0 & -185.65 & 0.2 & 0.4 \\
HH para & -121.2 & -120.59 & 0.5 & 0.4 \\
${ }^{13} \mathrm{CH}$ & -2689.7 & -2901.46 & -7.3 & -5.5 \\
\hline
\end{tabular}

a Difference, in per cent, of first two columns.

${ }^{b}$ Corrections for vibrations, in per cent, calculated from the model discussed in the text.

One way to obtain some estimate of the magnitude of the vibrational corrections is the study of a molecule of known geometry. We have done this for benzene. From high precision frequency measurements of a number of spectrum peaks, experimental values for the dipolar couplings were obtained; they are given in the first column of Table 1. Calculated values, given in the second column, were obtained assuming a rigid hexagonal arrangement of the protons and $\mathrm{H}-\mathrm{H}$ and $\mathrm{C}-\mathrm{H}$ distances of 2.47 and $1.08 \AA$, respectively; the ortho coupling was taken equal to the experimental one, as lack of knowledge of the orientational constant allows calculation of ratios of couplings only. The third column gives the per cent difference of the first two columns. This difference is presumably due to the combined influence of vibrations and of anisotropy of the indirect interactions. We shall for the moment disregard the latter; it will be discussed in the next section.

A very simple model to estimate the contribution of the vibrations has been suggested in reference 4 . In this model, only the $\mathrm{C}-\mathrm{H}$ bond stretching and bending is considered, the heavier carbon atoms being taken as a fixed framework. On the basis of this model the differences given in the fourth column of Table 1 have been calculated. Considering the assumptions made, the agreement is as good as can be expected. Although generalizations from 
this simple case are hazardous, it is our hope that simple corrections, taking into account the vibrations of the hydrogen atoms only, may prove to be of reasonable accuracy.

\section{THE ANISOTROPY OF THE INDIRECT SPIN-SPIN COUPLING}

Anisotropy of the indirect spin-spin coupling contributes terms in the spin Hamiltonian which have the same form as those for the direct dipolar coupling ${ }^{7}$, and therefore cannot be separated from them in a regular spectrum analysis. We shall refer to these terms as the pseudo-dipolar coupling. As the direct coupling is needed for an interpretation in terms of a molecular geometry, an estimate of the pseudo-dipolar contribution is important. Reference 2 gives a formal treatment of these contributions.

Table 2. Indirect spin-spin couplings in $\mathrm{CH}_{3} \mathrm{~F}$ (in hertz).

\begin{tabular}{crrr}
\hline Type & \multicolumn{1}{c}{$\Delta J^{\text {calc } a}$} & \multicolumn{1}{c}{$J^{\text {calc }}$} & \multicolumn{1}{c}{$J^{\text {exp }}$} \\
\hline C-F & 263.6 & -97.5 & -161.9 \\
C-H & 4.7 & 172.8 & 148.8 \\
H-F & -107.1 & 66.6 & 46.3 \\
H-H & -8.9 & -30.0 & -9.6 \\
\hline
\end{tabular}

${ }^{a} \Delta J=J_{z z}-\frac{1}{2}\left(J_{x x}+J_{y y}\right)$, where $z$ is the direction of the three-fold axis.

Gerritsen and MacLean ${ }^{8}$ have estimated the relative importance of direct and indirect contributions from studies of the same compound in different liquid crystalline solvents. But a more promising approach is the theoretical calculation of the anisotropy of the indirect interactions. Such calculations have been published by a number of authors ${ }^{9-12}$. All these calculations have been based on semiempirical methods, and the results have varied quite widely. Recently Ditchfield and Snyder ${ }^{13}$ have made $a b$ initio calculations for methyl fluoride. Their main results are summarized in Table 2. The first and second columns give, respectively, the calculated anisotropic and isotropic parts of the indirect coupling (cylindrical symmetry around the bond is assumed). The last column gives experimental values for the isotropic coupling, and will give an idea of the accuracy of the calculations. In considering the magnitude of the anisotropic part of the coupling, it must be remembered that it must be multiplied by a factor smaller than the orientational constant in order to obtain the pseudo-dipolar term. In methyl fluoride this factor is about 0.01. Thus it is seen that the pseudo-dipolar coupling is negligible for $\mathrm{H}-\mathrm{H}$ and $\mathrm{C}-\mathrm{H}$ couplings, and quite small for $\mathrm{H}-\mathrm{F}$ and $\mathrm{C}-\mathrm{F}$ couplings. We believe it probable that this will be generally true.

\section{CONCLUSIONS}

In the deuterium substitution method, which has been described for cyclohexane- $\mathrm{d}_{11}$, the fully protonated position provides a viewpoint from 


\section{NMR IN LIQUID CRYSTALLINE SOLVENTS}

which distances to other protons, distributed at random through the molecule, can be surveyed. One could envision the preparation of a number of deuterated species of a molecule, each species having a different, well-chosen, fully protonated site. These sites would then provide a number of complementary viewpoints of the molecule, which in combination may give a wealth of structural information.

We are hopeful that simple models will give an account of the major effect of vibrations. Recent theoretical work suggests that for hydrocarbons pseudo-dipolar interactions will not significantly hinder molecular structure determination.

\section{REFERENCES}

1 A. Saupe and G. Englert. Phys. Rev. Letters 11. 462 (1963).

2 A. Saupe, in Magnetic Resonance, Coogan et al., eds. p. 339, Plenum Press, New York (1970).

${ }^{3}$ P. Diehl and C. L. Khetrapal, NMR, Basic Principles and Progress, Vol. 1. Springer, New York (1969).

${ }^{4}$ S. Meiboom and L. C. Snyder, Accounts of Chemical Research 4, 81 (1971).

5 R. C. Hewitt, Rev. Scient. Instr. 39, 1066 (1968).

6 A. L. Bloom and J. N. Shoolery, Phys. Rev. 97, 1261 (1955).

7 L. C. Snyder, J. Chem. Phys. 43, 4041 (1965).

8 J. Gerritsen and C. MacLean, Molecular Crystals and Liquid Crystals 12, 97 (1971).

9 M. Barfield, Chem. Phys. Letters 4, 518 (1970).

${ }^{10}$ H. Nakatsuji, H. Kato, I. Morishima and T. Yonezawa, Chem. Phys. Letters 4, 607 (1970).

11 A. D. Buckingham and I. Love, J. Magn. Resonance 2, 338 (1970).

12 H. Nakatsuji. K. Hirao. H. Kato and T. Yonezawa, Chem. Phys. Letters 6, 541 (1970).

13 R. Ditchfield and L. C. Snyder, 56. 5823 (1972). 